\title{
COMPARISON OF THE EFFECTS OF THYME AND OREGANO ON HEMATOLOGY IN LAYING JAPANESE QUAIL
}

\section{(C) Aghdam Shahryar Habib, Ebrahimnezhad Yahya, Gholipoor Vahid}

Department of Animal Science, Shabstar branch, Islamic Azad University, Shabstar, Iran, Email: ha_shahryar@yahoo.com

The aim of this study was to compare effects of oregano (Mentha pulegiym), thyme (Thymus) on immune system in laying Japanese quail. 420 quails were feed in completely random design by 7 treatments and 4 replications (every replication involves 12 female and 3 male quails) by following ration for 45 days: 1) control diet (without thyme and oregano), 2)control diet+thyme $1.5 \%, 3$ )control diet+thyme 3\%, 4)control diet+thyme $4.5 \%, 5)$ control diet+oregano $1.5 \%$, 6 )control diet+oregano $3 \%, 7)$ control diet+oregano $4.5 \%$. At the end of the experiment, 2 birds were selected from each replication and the hematology measures were analyzed. The result showed that us- ing different levels of oregano and thyme in quail diet were affected on hematology. Monocytes had the highest consumer $4.5 \%$ oregano than control group (9.25 vs $6.5 \%$ ). Adding oregano until $4.5 \%$ had significant effect on basophile, oezinophil and monocyte. Intake level of $4.5 \%$ thyme observed highest level in basophile and oezinophil between groups (2.22 and 5.75 respectively). Containing $4.5 \%$ oregano in group, suggests that differences in antioxidant and antioxidation properties, thyme and oregano. In conclusion, add thyme and oregano to the $4.5 \%$ of the diet has significantly positive effect on the percentage of basophile, oezinophil and monocytes.

\section{MULTI-BIOACTIVE METABOLITES FROM RUDBECKIA HIRTA L. FLOWERS}

\section{(C) Ahmed Atallah F. ${ }^{1,2}$, Michael Botros R. ${ }^{2}$, Gedara Sahar R. ${ }^{2}$, Amer M. M. ${ }^{2}$, Stevenson Lesley ${ }^{3}$}

${ }^{1}$ Department of Pharmacognosy, College of Pharmacy, King Saud University, P. O. Box 2457, Riyadh 11451, Kingdom of Saudi Arabia

${ }^{2}$ Department of Pharmacognosy, Faculty of Pharmacy, Mansoura University, Egyp

${ }^{3}$ Centre for Phytochemistry and Pharmacology, Southern Cross University, PO Box 157, Lismore NSW 2480, Australia

Black-eyed Susan or Rudbeckia hirta L. (Asteraceae) is a popular garden biennial herb. American Indian used the root tea to treat worms and colds, and as a wash for sores and snakebites, while the root juice has been used to treat earache (1). The immunomodulatory organic extract of $R$. hirta flower heads was partitioned and subjected fractionation using a series of chromatographic techniques which led to the isolation of a new highly oxygenated pseudoguaianolide: $\left(1 S^{\star}, 4 S^{\star}, 5 R^{\star}, 6 R^{\star}, 7 S^{\star}\right.$, $\left.10 S^{\star}, 11 S^{\star}\right)-4,14,15$-tri-acetoxy-pseudoguaian-12,6-olide along with three phenolic acids: $\beta$-resorcylic acid, (E)-p-coumaric acid, and (E)-caffeic acid; two phenolic esters: 3-O- (E)-caffeoylquinic acid and 3-O(E)-coumaroylquinic acid methyl ester; a phenolic acid ether (Z)-p-coumaric acid-4-O- $\beta$-D-glucopyranoside; two flavonol glycosides: gossypitrin and quercetagitrin; three methylated flavonol glycosides: eupatolin, patulitrin, eupatolitin-3-O- $\beta$-D-glucopyranoside; and eupatolitin-3-methyl ether. The structure of the new sesquiterpene lactone 1 was established on the basis of extensive spectroscopic analyses, including 1D and 2D NMR. Most of isolated compounds exhibited antioxidant (oxygen radical absorbance capacity, ORAC), immunomodulatory, 5-lipoxygenase (5-LOX) inhibitory and cytotoxic activities at variable concentrations, which can be considered as a partial scientific evidence for the ethnopharmacological uses of the plant.

Reference: Barker, J. (2004) "The Encycolpedia of North American Wild Flowers". Parragon Publishing Ltd. Bath, UK. 